\title{
Guest editorial: special issue on selected papers from the European conference on genetic programming
}

\author{
Sara Silva $\cdot$ James A. Foster
}

Published online: 13 May 2012

(C) Springer Science+Business Media, LLC 2012

The European Conference on Genetic Programming (EuroGP) is the only conference worldwide exclusively devoted to Genetic Programming (GP) and the evolutionary generation of computer programs. Similarly, Genetic Programming and Evolvable Machines is the academic journal with the strongest focus on the subject. So it is particularly fitting to have this special issue of GPEM dedicated to expanded versions of the top papers from the 14th annual EuroGP. It is the goal of this special issue to raise awareness to the quality and diversity of the research being presented at EuroGP.

EuroGP began as a way to support the international GP research community in a European venue. In 1995, GP researchers at the sixth International Conference on Genetic Algorithms (ICGA) in Champaign, Illinois, USA, discussed whether GP needed a more focused event, given the rapid maturation of the field. In response, in 1996, John Koza (together with David Goldberg, David Fogel, and Rick Riolo) organized the first Genetic Programming conference at Stanford University. Around this time, leaders in the GP community, including Wolfgang Banzhaf and William Langdon, names familiar to GPEM readers, began discussing the need for a similar conference in Europe, since much of the GP work was being done in Europe while most of the GP-friendly conferences were in the USA. Thus was born the first EuroGP workshop in 1998 in Paris, France. In the meantime, in 1999 in Orlando Florida, USA, the International Conference on Genetic Algorithms (ICGA) and the GP conference merged to form the Genetic and Evolutionary Computation

\section{S. Silva $(\bowtie)$}

INESC-ID Lisboa, Instituto Superior Tecnico, Universidade Tecnica de Lisboa, Rua Alves Redol 9 , 1000-029 Lisboa, Portugal

e-mail: sara@kdbio.inesc-id.pt

\section{J. A. Foster}

Biological Sciences and Institute for Bioinformatics and Evolutionary STudies (IBEST),

University of Idaho, Moscow, ID 83844-0351, USA

e-mail: foster@uidaho.edu 
Conference (GECCO), which has met annually ever since. GECCO met only in the USA until 2007 when it met in London, England. The EuroGP workshop continued to meet annually. At the 2000 meeting in Edinburgh, Scotland, EuroGP transformed into a conference, designed to maintain the intimacy of a workshop with a single track promoting informal interactions between participants. The first non-European co-chair of EuroGP, James A. Foster from Idaho, USA (one of the editors of this special issue) led the meeting, together with Evelyne Lutton from Paris, France. Since then, EuroGP has met in every corner of Europe, from Gothenburg, Sweden to Naples, Italy; and from Coimbra, Portugal to Istanbul, Turkey; and at many lovely points in between. EuroGP has maintained its independent status and workshop atmosphere, though it has co-located since 2007 with other workshops and conferences focused on specific areas of evolutionary computation research, at an annual event known as EvoStar.

The 14th EuroGP, from which the papers in this special issue were selected, took place 27-29 April, 2011, in Torino, Italy. Even though funding for conference participation has become scarcer each year, EuroGP-2011 had a $25 \%$ increase in the number of submissions from the preceding year. We received 59 submissions, from 25 different countries across four continents. These were reviewed by an international Programme Committee composed of 79 reviewers. Finally, 29 papers were accepted for inclusion in the proceedings (49\%), 20 of them for oral presentation during the conference (34\%); 7 of these were nominated for the EuroGP Best Paper Award. From these best paper nominees, the 4 papers that were rated highest by the reviewers and editors were selected and their authors invited to submit an extended version of their work to this special issue. These papers went through an extensive review process. Besides being re-reviewed by some members of the EuroGP programme committee, additional GPEM reviewers also contributed to ensure these extended versions meet the highest scholarly standards.

The first paper introduces a new form of Linear GP (LGP) called Parallel LGP (PLGP) for classification problems. Based on the usage of multiple lists of instructions executed in parallel, PLGP is able to limit the disruptive effects of crossover and mutation, significantly outperforming regular LGP. It also decreases fitness evaluation time by using a novel caching technique developed specifically for PLGP.

The second paper is a study of evolutionary dynamics aimed at understanding the role of neutrality in GP, a contentious topic in the community. A measure of robustness is used to quantify neutrality, and the authors analyse the interplay between robustness, evolvability, and accessibility amongst genotypes, phenotypes, and fitness values in Linear Genetic Programming.

The third paper describes the Regulatory Network Computational Device, a new variant of the successful Artificial Gene Regulatory Network (ARN) model. These models explore the new biological knowledge of regulatory mechanisms that are fundamental in both processes of inheritance and of development in natural systems, including those mechanisms in evolutionary algorithms by using a different than usual genotype-phenotype mapping.

Finally, the fourth paper describes an interesting application where GP is used to evolve brain-computer interfaces for mouse control. Electrical brain signals are 
analysed and directly transformed into pointer movements, the only input provided being the set of visual stimuli to be used to generate recognisable brain activity. The results provided by GP are better than those produced by support vector machines.

We look forward to continuing the tradition represented in this special issue of GPEM. EuroGP-2012 has recently been held in Malaga, Spain, and we encourage readers to attend EuroGP-2013 in Vienna, Austria, and to submit their best work for presentation.

The guest editors thank all the authors who submitted their work to the 14th EuroGP, and all the reviewers who dedicated their time and scientific expertise to ensure the high quality of the papers presented at the conference and the ones included in this special issue. A heartfelt thanks goes to Jennifer Willies from Edinburgh Napier University. She is the soul of EuroGP and EvoStar, and we can truthfully state that without her dedicated work each and every year since 1998, EuroGP would not be such a successful and pleasant conference. We also want to thank Springer for accompanying EuroGP from the very beginning. Finally, we would like to thank Lee Spector for his openness, help and encouragement to this project, and Geetha Subramanian of Springer for all her helpfulness during this process. 